\title{
The correlation between solar and geomagnetic activity - Part 2: Long-term trends
}

\author{
Z. L. Du \\ Key Laboratory of Solar Activity, National Astronomical Observatories, Chinese Academy of Sciences, Beijing 100012, \\ China
}

Received: 15 January 2011 - Revised: 13 July 2011 - Accepted: 18 July 2011 - Published: 5 August 2011

\begin{abstract}
Using lag-correlation function analysis, the correlation coefficient at zero lag $\left(r_{0}\right)$, the maximum $\left(r_{\mathrm{m}}\right)$ and the corresponding lag time $\left(L_{\mathrm{m}}\right)$ between solar $\left(R_{\mathrm{z}}\right)$ and geomagnetic $(a a)$ activity for a 528-month (44-year) running time window are shown to vary in a declining, declining and rising secular trend, respectively, before 1958. However, these trends changed since 1958 with a rising secular trend in both $r_{0}$ and $r_{\mathrm{m}}$ and without a significant trend in $L_{\mathrm{m}}$, probably related to a periodicity longer than 140 years. An odd-numbered solar cycle tends to show a higher correlation and a shorter lag time between $R_{\mathrm{z}}$ and $a a$ than the previous even-numbered one, suggesting a 2-cycle periodicity superimposed on secular trends. An even-numbered Hale cycle tends to show a higher correlation and a shorter lag time between $R_{\mathrm{Z}}$ and $a a$ than the previous odd-numbered one, suggesting a 4-cycle periodicity superimposed on secular trends. The variations in the correlations may be related to the nonlinearity between $R_{\mathrm{z}}$ and $a a$, and the decreasing trend in the correlation $\left(r_{0}\right)$ is not exclusively caused by the increasing trend in the lag time of $a a$ to $R_{\mathrm{z}}$. These results represent an observational constraint on solar-dynamo models and can help us gain a better understanding of the long-term evolution of solar activities. In applications, therefore, cautions must be taken when using the correlation for molding the dynamical process of the Sun and for predicting solar activities.
\end{abstract}

Keywords. Geomagnetism and paleomagnetism (Time variations, secular and long term)

\section{Introduction}

The aa geomagnetic index, calculated from the 3-hourly $\mathrm{K}$ indices measured at two near-antipodal midlatitude stations (Mayaud, 1972), has been used for analyzing long-term

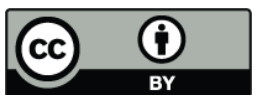

Correspondence to: Z. L. Du

(zldu@nao.cas.cn) trends in the global geomagnetic activity and in its correlation with solar activity (Feynman, 1982; Legrand and Simon, 1989; Mursula et al., 2004; Lukianova et al., 2009). It has an 11-year variation similar to that of solar activity, as described by the Zürich relative sunspot number $\left(R_{\mathrm{Z}}\right)$. In the twentieth century, there has been a significant increase in the $a a$ index, the reason for which, however, is unknown (Feynman and Crooker, 1978; Clilverd et al., 1998; Demetrescu and Dobrica, 2008; Lukianova et al., 2009).

Studying the correlation between the $a a$ and $R_{\mathrm{z}}$ series is useful for understanding the long-term evolution of solar activity (Legrand and Simon, 1989; Russell and Mulligan, 1995; Prestes et al., 2006; Cameron and Schüssler, 2007). Borello-Filisetti et al. (1992) examined the secular variations in the correlation between $a a$ and $R_{\mathrm{Z}}$ in terms of ascending (A) and descending (D) phases of the solar cycle and pointed out that the linear correlation coefficient $(r)$ during the Dphase tends to decrease. They also suggested by visual inspection two periodicities of 5-cycle in A-phase, which they insist on later (Mussino et al., 1994), and 8-cycle in D-phase. Kishcha et al. (1999) examined the long-term variations in the 23-year running correlation and suggested that the decreasing trend of the correlation is caused by the upward linear trend of the time delay of $a a$ to $R_{\mathrm{z}}$ accompanied by a quasi-periodicity of 40-50 years. Echer et al. (2004) suspected that the long-term decrease in the correlation has a monotonic nature or it is part of a long solar activity periodicity. We (Du et al., 2009; Du and Wang, 2011) studied the geomagnetic precursor prediction method and found that its predictive power shows a weakening trend and a cyclical behavior of about 44 years. Therefore, studying the variations in the correlation between $a a$ and $R_{\mathrm{Z}}$ is useful for understanding the solar dynamo theory, in which the level of geomagnetic activity in the declining phase of a solar cycle is related to the magnitude of the maximum solar activity in the ensuing cycle (Schatten et al., 1978).

Firstly, in this paper, we present some phenomena related to the correlation between $R_{\mathrm{Z}}$ and $a a$. Long-term trends in

Published by Copernicus Publications on behalf of the European Geosciences Union. 

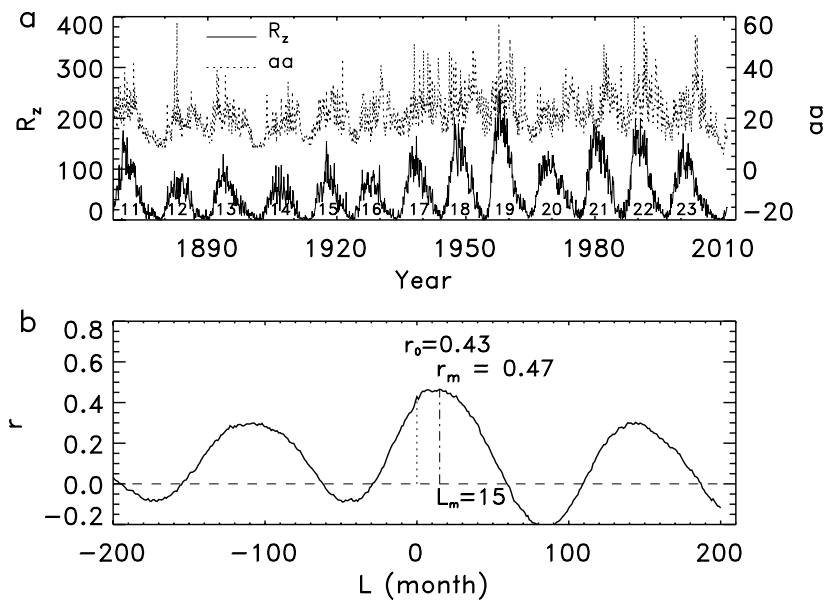

Fig. 1. (a) Monthly $R_{\mathrm{Z}}$ (solid) and $a a$ (dotted). (b) Correlation function between $R_{\mathrm{Z}}$ and $a a$ of the lag $L=-200,-199, \ldots, 200$ (month).

the correlation between $R_{\mathrm{Z}}$ and $a a$ are statistically studied with a 528-month (44-year) running time window using lagcorrelation analysis in Sect. 2. The correlations between $R_{\mathrm{Z}}$ and $a a$ for the 11-year Schwabe cycle and the 22-year Hale cycle are analyzed in Sects. 3 and 4, respectively. The results are briefly discussed and summarized in Sect. 5. And then, in the following paper ( $\mathrm{Du}, 2011 \mathrm{~b}$ ), we will present a model to explain these phenomena and the significant increase in the $a a$ index (and its baseline) over the twentieth century.

\section{Correlation analysis for a 528-month running time window}

The reliable monthly $a a$ index is only available since 1868 (Mayaud, 1972). Nevanlinna and Kataja (1993) extended the $a a$-index series back to 1844 from measurements taken in Finland (Helsinki). However, the data available before 1868 are only yearly values, so we use the monthly $a a$ index ${ }^{1}$ and relative sunspot number ${ }^{2}\left(R_{\mathrm{Z}}\right)$ from January 1868 to October 2010. Recently, the $a a$ index was suggested to have an error and should be increased by $3 \mathrm{nT}$ before 1957 (Nevanlinna and Kataja, 1993; Svalgaard et al., 2004; Lukianova et al., 2009). The corrected $a a$ series is used in the present study and the original uncorrected $a a$ series is discussed at last. There are 1714 data pairs in all, as plotted in Fig. 1a.

Firstly, we use all the data to calculate the (linear) correlation function $(r)$ between $R_{\mathrm{z}}$ and $a a$ of the lag $L=$ $-200,-199, \ldots, 200$, as shown in Fig. 1b. One can see that $r$ varies with a periodicity of about 130 months, which represents the ( $\sim 11$-year) Schwabe solar cycle in both $R_{\mathrm{z}}$ and $a a$. Most of the values are positive (e.g. for $-29 \leq L \leq 59$ when

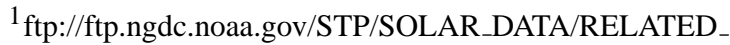
INDICES/AA_INDEX/

${ }^{2}$ http://www.ngdc.noaa.gov/stp/spaceweather.html
}

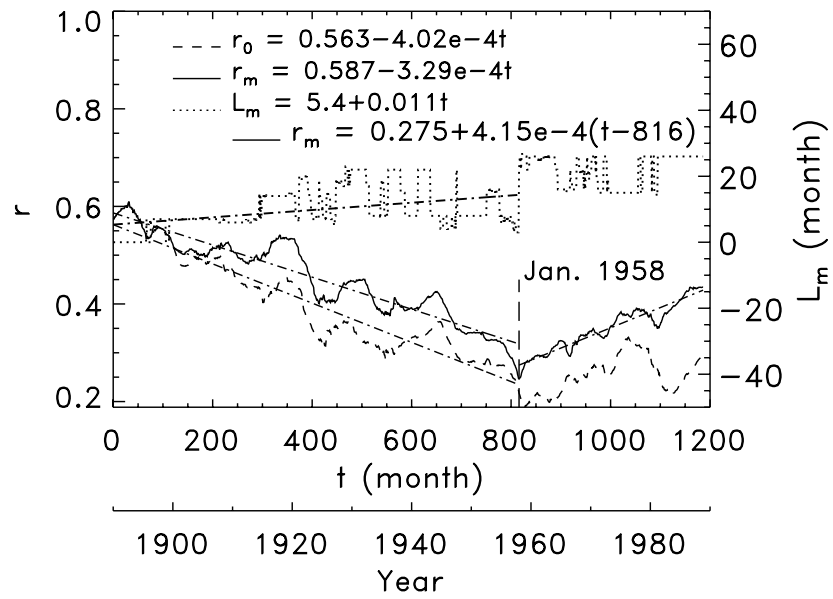

Fig. 2. Trends of $r_{0}$ (dashed), $r_{\mathrm{m}}$ (solid) and $L_{\mathrm{m}}$ (dotted) for a 528-month running time window.

$R_{\mathrm{Z}}$ and $a a$ have nearly the same phase), and a few other values are negative (e.g. for $-61 \leq L \leq-30$ and $60 \leq L \leq 109$ when $R_{\mathrm{z}}$ and $a a$ have nearly the opposite phase), implying that $r$ is asymmetrical. The $r$-coefficient at $L=0$ is $r_{0}=0.43$, and the maximum one is $r_{\mathrm{m}}=0.47$ at a lag of $L_{\mathrm{m}}=15$. It means that $R_{\mathrm{z}}$ and $a a$ are well correlated at the $99 \%$ level of confidence but that the latter lags behind the former by about 15 months - the solar activity is at the origin of the geomagnetic activity. The $r$-coefficients will improve greatly $\left(r_{0}=0.58, r_{\mathrm{m}}=0.66\right)$ if using the 13-month running means of $R_{\mathrm{z}}$ and $a a$.

Now, we study the long-term trends of $r_{0}, r_{\mathrm{m}}$ and $L_{\mathrm{m}}$ in terms of a running time window of $w=528$ months (44year), similar to those as did by Kishcha et al. (1999). For each time window $(t=0,1, \ldots, 1186)$, we calculate the correlation function between $R_{\mathrm{Z}}(i)$ and $a a(i+L)$ of the lag $L=-50,-49, \ldots, 70$ (similar to Fig. 1b) for $i=t, t+1, \ldots$, $t+w-1$, and select the $r$-coefficient at $L=0\left(r_{0}\right)$, the maximum value $\left(r_{\mathrm{m}}\right)$ and its corresponding lag $\left(L_{\mathrm{m}}\right)$. At the two ends of the data, the amounts of data are suitably adjusted to ensure that they are not out of the observational ranges. The results are shown in Fig. 2.

It can be clearly seen in Fig. 2 that the correlation between $R_{\mathrm{Z}}$ and $a a\left(r_{0}\right.$, dashed) varies apparently in a declining trend before 1958, though with fluctuations, from about 0.6 in 1890 s to about 0.2 near 1958. A linear (dash-dotted through the dashed) line is also shown in the figure, with its least-squares-fit regression equation given by

$r_{0}=0.563 \pm 0.002-(4.02 \pm 0.04) \times 10^{-4} t$,

where $t$ is in units of months labeled from January 1890 (centered date of the first window), and \pm represents the standard deviation. The standard deviation of the regression equation is $\sigma_{0}=0.029$. The correlation coefficient between the fitted and original values is $r_{\mathrm{f}}=0.96$ at a confidence level (CL) greater than $99 \%$. It suggests that $a a$ was not correlated with 
$R_{\mathrm{Z}}$ in the same way for different periods of time, and that $r_{0}$ varied in a weakening trend over time (Kishcha et al., 1999; Echer et al., 2004). Similarly, the maximum correlation coefficient $\left(r_{\mathrm{m}}\right.$, solid) also varies in a declining trend,

$r_{\mathrm{m}}=0.587 \pm 0.002-(3.29 \pm 0.04) \times 10^{-4} t$,

with a standard deviation of $\sigma_{\mathrm{m}}=0.026$ and a fitting correlation coefficient of $r_{\mathrm{f}}=0.95$. The corresponding lag $\left(L_{\mathrm{m}}\right.$, dotted) varies roughly in a rising trend (Kishcha et al., 1999),

$L_{\mathrm{m}}=5.4 \pm 0.4+(0.011 \pm 0.001) t$,

with a standard deviation of $\sigma_{\mathrm{L}}=5.8$. However, the rising trend in $L_{\mathrm{m}}$ is very weak $\left(r_{\mathrm{f}}=0.41\right)$ and there is not an apparent trend in $L_{\mathrm{m}}$ since 1958.

The anti-correlation between $L_{\mathrm{m}}$ and $r_{0}$ or $r_{\mathrm{m}}$ means that the more the geomagnetic activity lags behind the solar activity, the weaker the correlation. Kishcha et al. (1999) suggested that it is the variations of time delay $\left(L_{\mathrm{m}}\right)$ that cause the variations of the solar-geomagnetic correlation $\left(r_{0}\right)$. Nevertheless, the correlation $r_{\mathrm{m}}$ still decreases even if the time delay is considered, as shown (solid line) in Fig. 2.

If these trends continue, the value of $r_{\mathrm{m}}\left(r_{0}\right)$ will become smaller over time. However, $r_{\mathrm{m}}\left(r_{0}\right)$ seems to behave differently on two sides of the year 1958 (peak of Cycle 19 and peak of a possible Gleissberg 90-year cycle): a declining trend before and a rising trend after 1958. For example, $r_{\mathrm{m}}$ varies in a rising trend since 1958 ,

$r_{\mathrm{m}}=0.275 \pm 0.001+(4.15 \pm 0.06) \times 10^{-4}(t-816)$.

with a standard deviation of $\sigma_{\mathrm{m}}^{\prime}=0.013$ and a fitting correlation coefficient of $r_{\mathrm{f}}^{\prime}=0.96$. It implies that the correlations cannot decrease infinitely and that they may vary cyclically in a periodicity longer than 140 years, that is, double the time period from before 1890 to 1958 , which may be related to a possible double Gleissberg cycle or the 200-year Suess cycle. If it turns out to be true that the year 1958 is a turning point for the correlations, they should increase from that time in long-term trends, which should be analyzed with more data in the future.

\section{Correlation analysis for solar cycles}

In the previous section, we have used a running time window to analyze the successive variations in the correlation between $R_{\mathrm{z}}$ and $a a$. Now, we study the behavior of the correlation in terms of the ascending phase (A), the descending phase (D) and the 11-year (Schwabe) solar cycle $(n)$. For the A-phase in each Cycle $n=11,12, \ldots, 23$, we calculate the correlation function between $R_{\mathrm{z}}(i)$ and $a a(i+L)$ of the lag $L=-50,-49, \ldots, 70$ (similar to the technique in Figs. 12) for $i=m(n), m(n)+1, \ldots, M(n)$, where $m(n)$ is the date of sunspot minimum between Cycles $n-1$ and $n$, and $M(n)$ is the date of maximum in Cycle $n$ (Borello-Filisetti et al., 1992; Du and Du, 2006). Then we select the $r$-coefficient at
$L=0\left(r_{\mathrm{A} 0}\right)$, the maximum value $\left(r_{\mathrm{Am}}\right)$ and its corresponding lag $\left(L_{\mathrm{Am}}\right)$. The values of the eleventh cycle are deleted for short data. Similarly, for the D-phase, we calculate the correlation function between $R_{\mathrm{z}}(i)$ and $a a(i+L)$ for $i=M(n)$, $M(n)+1, \ldots, m(n+1)$, and select the $r$-coefficient at $L=0$ $\left(r_{\mathrm{D} 0}\right)$, the maximum value $\left(r_{\mathrm{Dm}}\right)$ and its corresponding lag $\left(L_{\mathrm{Dm}}\right)$. Finally, for each Cycle $n$, we calculate the correlation function between $R_{\mathrm{Z}}(i)$ and $a a(i+L)$ for $i=m(n), m(n)+1$, $\ldots, m(n+1)$ and select the $r$-coefficient at $L=0\left(r_{\mathrm{n} 0}\right)$, the maximum value $\left(r_{\mathrm{nm}}\right)$ and its corresponding lag $\left(L_{\mathrm{nm}}\right)$. At the two ends of the data, suitable adjustments were also made to ensure that they are within their ranges. The results are shown in Fig. 3.

For the A-phase in Fig. 3a, $r_{\mathrm{A} 0}$ (dashed) shows a weak declining trend with cycle number $n$, as suggested by BorelloFilisetti et al. (1992), $r_{\text {Am }}$ (solid) changes little, and $L_{\mathrm{Am}}$ (dotted) varies in a weak rising trend. Their linear regression equations are given by

$$
\begin{aligned}
& r_{\mathrm{A} 0}=0.83 \pm 0.19-(0.020 \pm 0.011) n, \\
& r_{\mathrm{Am}}=0.69 \pm 0.12-(0.002 \pm 0.007) n, \\
& L_{\mathrm{Am}}=-26 \pm 30+(2.0 \pm 1.7) n,
\end{aligned}
$$

with the fitting correlation coefficients of $r_{\mathrm{f}}=0.50,0.09$ and 0.34 , respectively, but all three statistically insignificant at the $95 \%$ level of confidence (CL of $90 \%, \sim 0$ and $71 \%$ ). The standard deviations (of the regression equations) are $\sigma=0.12,0.08$ and 19, respectively. The values of $r_{\mathrm{A} 0}$ and $r_{\text {Am }}$ are all positive. The $L_{\text {Am }}$ values are negative about -9 months for Cycles 13-15 and positive in other cycles, with a maximum value of 60 months for Cycle 18. The 5-cycle periodicity in $r_{\text {A0 }}$ suggested by Borello-Filisetti et al. (1992) has not been found in Fig. 3a.

For the D-phase in Fig. 3b,

$r_{\mathrm{D} 0}=0.84 \pm 0.43-(0.035 \pm 0.025) n$,
$r_{\mathrm{Dm}}=0.70 \pm 0.18-(0.004 \pm 0.010) n$,
$L_{\mathrm{Dm}}=-13 \pm 12+(2.0 \pm 0.7) n$,

with the standard deviations of $\sigma=0.32,0.13$ and 9.0 , respectively. The declining trends in $r_{\mathrm{D} 0}$ and $r_{\mathrm{Dm}}$ are very weak $\left(r_{\mathrm{f}}=0.39,0.13\right)$ and statistically insignificant at the $95 \%$ level of confidence. The $r_{\mathrm{D} 0}$ values are negative for Cycles 17-18 and 20-21 with a negative maximum $(-0.36)$ for Cycle 20, which is due to the fact that the a minimum in this cycle occurs after rather than before the timing of the peak of $R_{\mathrm{Z}}$ (Fig. 1). The 8-cycle periodicity in $r_{\mathrm{D} 0}$ suggested by Borello-Filisetti et al. (1992) has not been found as well. In contrast, $L_{\mathrm{Dm}}$ shows an apparent rising trend with a fitting correlation coefficient of $r_{\mathrm{f}}=0.66$ significant at the $98 \%$ level of confidence.

For the solar cycle in Fig. 3c,

$$
\begin{aligned}
& r_{\mathrm{n} 0}=0.87 \pm 0.27-(0.032 \pm 0.02) n, \\
& r_{\mathrm{nm}}=0.68 \pm 0.17-(0.010 \pm 0.010) n, \\
& L_{\mathrm{nm}}=-17 \pm 18+(2.2 \pm 1.1) n,
\end{aligned}
$$



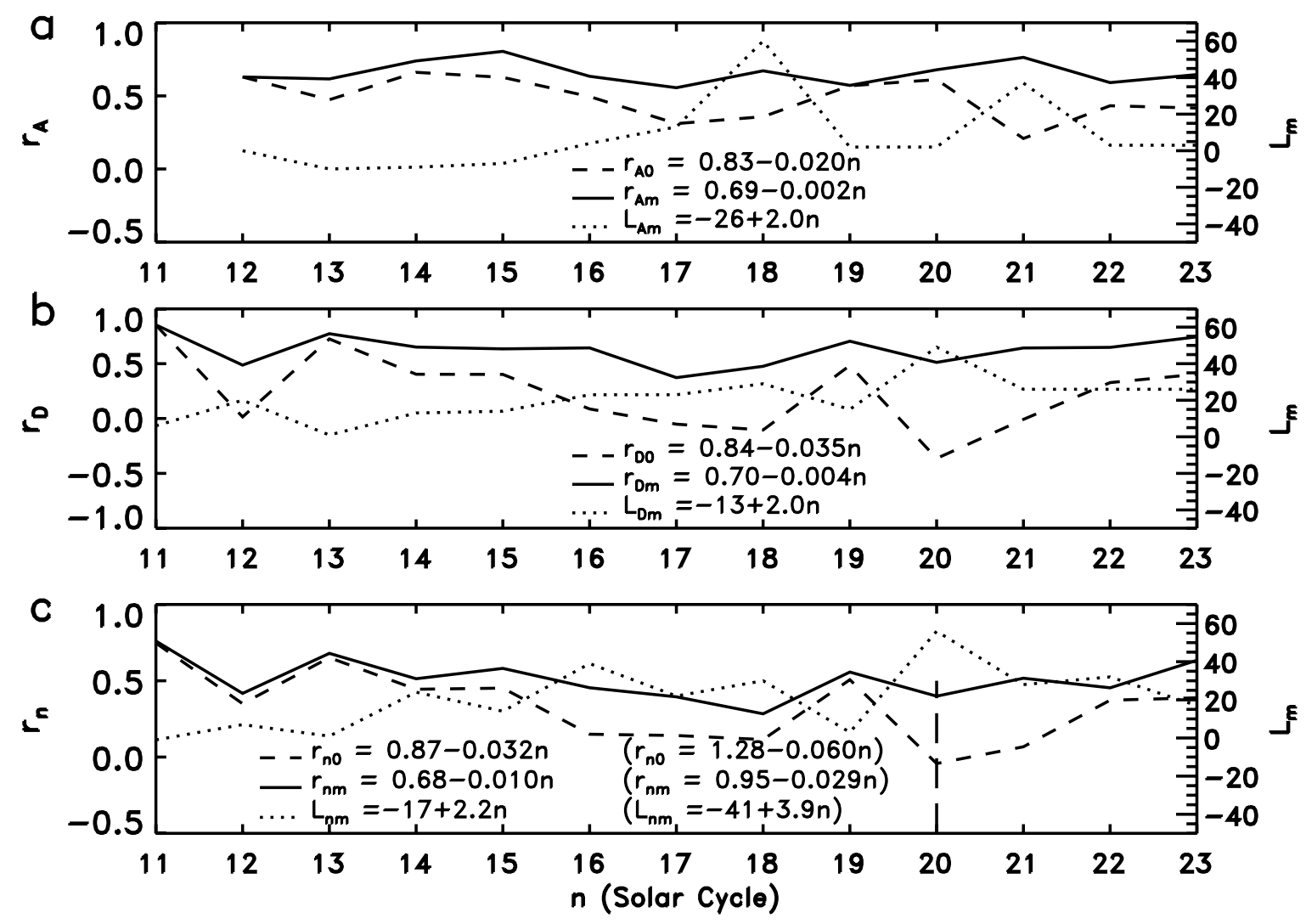

Fig. 3. Trends of $r_{0}$ (dashed), $r_{\mathrm{m}}$ (solid) and $L_{\mathrm{m}}$ (dotted) for A-phases (a), D-phases (b), and for solar cycles (c).

with the standard deviations of $\sigma=0.20,0.13$ and 14.1 , and the fitting correlation coefficients of $r_{\mathrm{f}}=0.52,0.29$ and 0.53 , respectively, but all three statistically insignificant at the $95 \%$ level of confidence (CL of $93 \%, 65 \%$ and $93 \%$ ).

Similar to Fig. 2, the trends in Fig. $3 \mathrm{c}$ changed after about Cycle 20: the declining trend in $r_{\mathrm{n} 0}$ (dashed) before Cycle 20 changed to a rising trend after Cycle 20; the rising trend in $L_{\mathrm{nm}}$ (dotted) before Cycle 20 changed to a weak declining trend after Cycle 20. For the data before Cycle 20,

$$
\begin{aligned}
& r_{\mathrm{n} 0}=1.28 \pm 0.33-(0.0 .060 \pm 0.020) n, \\
& r_{\mathrm{nm}}=0.95 \pm 0.21-(0.029 \pm 0.013) n, \\
& L_{\mathrm{nm}}=-41 \pm 26+(3.9 \pm 1.6) n,
\end{aligned}
$$

with the standard deviations of $\sigma=0.18,0.12$ and 14.1, and the fitting correlation coefficients of $r_{\mathrm{f}}=0.71,0.60$ and 0.64 , respectively, all three statistically significant at around the $95 \%$ level of confidence (CL of $98 \%, 93 \%$ and $95 \%$ ).

The different behaviors on the two sides of Cycle 20 are similar to those in Fig. 2 and may be related to the increasing trend in $R_{\mathrm{Z}}$ before Cycle 20 (Fig. 1a) and a possible decreasing trend since then.

Besides weak long-term trends, an odd-numbered cycle tends to show a higher correlation $\left(r_{\mathrm{nm}}\right.$ or $\left.r_{\mathrm{n} 0}\right)$ and a smaller lag time ( $L_{\mathrm{nm}}$ ) of $a a$ to $R_{\mathrm{Z}}$ than the preceding even-numbered one, suggesting a periodicity of about 2 -cycle $(\sim 22$ years $)$ except for the even-odd cycle pair of $n=16-17$ which may be related to the different behaviors of sunspots on the two sides of Cycle 16 (Oliver et al., 1998; Duhau, 2003). In fact, these results are similar and related to the so-called G-O rule that an odd-numbered cycle tends to be stronger than the previous even-numbered one (Gnevyshev and Ohl, 1948; Wilson, 1988) and may be explained by an integral model (Du, 2011b).

\section{Correlation analysis for Hale cycles}

An even-numbered cycle is preferentially paired with the following odd-numbered one (Wilson, 1988), constituting a Hale cycle of even-odd cycle pair. The Hale cycle reflects the 22-year magnetic cycle of the Sun, having been found in both $R_{\mathrm{z}}$ and $a a$ (Chernosky, 1966; Russell and Mulligan, 1995). In this section, we study the behavior of the correlation between the original $R_{\mathrm{z}}$ and $a a$ series in terms of the Hale cycle $(H)$. For each $H$-cycle $(H=7,8, \ldots, 12)$, we calculate the correlation function between $R_{\mathrm{Z}}(i)$ and $a a(i+L)$ of the lag $L=-50,-49, \ldots, 70$ for $i=m(2 H-2), m(2 H-2)+1, \ldots$, $m(2 H)$, and select the $r$-coefficient at $L=0\left(r_{\mathrm{H} 0}\right)$, the maximum value $\left(r_{\mathrm{Hm}}\right)$ and its corresponding lag $\left(L_{\mathrm{Hm}}\right)$, as shown in Fig. 4. 


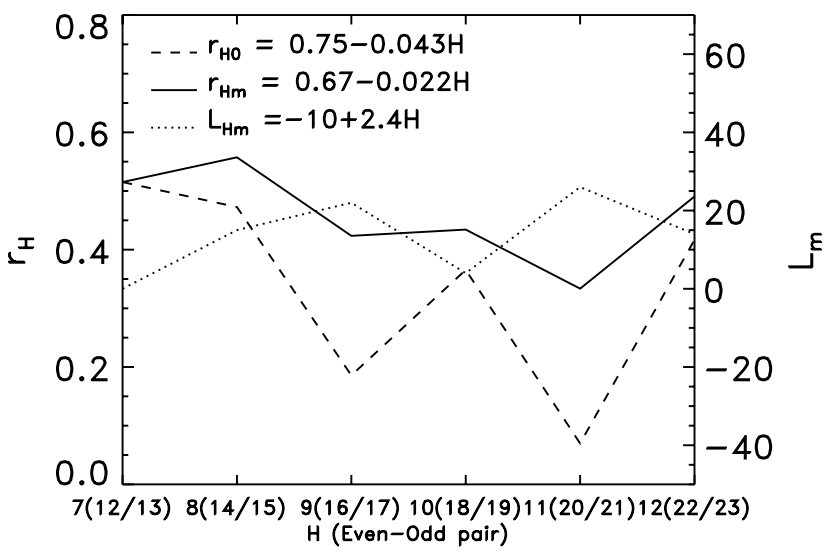

Fig. 4. Trends of $r_{\mathrm{H} 0}$ (dashed), $r_{\mathrm{Hm}}$ (solid) and $L_{\mathrm{Hm}}$ (dotted) for Hale cycles of E-O cycle pairs.

One can see in Fig. 4 that both $r_{\mathrm{H} 0}$ (dashed) and $r_{\mathrm{Hm}}$ (solid) vary in weak declining trends, while $L_{\mathrm{Hm}}$ (dotted) varies in a weak rising trend,

$r_{\mathrm{H} 0}=0.75 \pm 0.40-(0.043 \pm 0.041) H$,
$r_{\mathrm{Hm}}=0.67 \pm 0.17-(0.022 \pm 0.018) H$,
$L_{\mathrm{Hm}}=-10 \pm 23+(2.4 \pm 2.4) H$,

with the standard deviations of $\sigma=0.15,0.07$ and 8.9, respectively. However, these trends are very weak with the fitting correlation coefficients of $r_{\mathrm{f}}=0.47,0.53$ and 0.45 , at the $64 \%, 71 \%$ and $62 \%$ level of confidence, respectively.

Besides long-term trends, an even-numbered $H$-cycle tends to show a higher correlation $\left(r_{\mathrm{Hm}}\right.$ or $\left.r_{\mathrm{H} 0}\right)$ and a smaller lag time $\left(L_{\mathrm{Hm}}\right)$ of $a a$ to $R_{\mathrm{z}}$ than the neighboring oddnumbered $H$-cycle, suggesting a periodicity of about two Hale cycles ( $\sim 44$ years $)$, especially since $H=8$. If these behaviors continue, the correlation $\left(r_{\mathrm{H} 0}\right.$ or $\left.r_{\mathrm{Hm}}\right)$ should become weaker and the lag time $\left(L_{\mathrm{Hm}}\right)$ should become longer for the next even-odd cycle pair $(H=13$ for $n=24-25)$ than the present one $(H=12)$. These results may be related to the phenomena that an even-numbered $H$-cycle tends to be stronger than the previous odd-numbered one (Du, 2011b).

\section{Discussions and conclusions}

The correlation between the solar and geomagnetic activity $\left(R_{\mathrm{Z}}, a a\right)$ varies in a declining trend superimposed by some fluctuations, which has been re-analyzed. This study shows that the declining correlations (rising lag time) seem to have turned over (leveled off) since 1958 (Fig. 2): the declining trends in both $r_{0}$ and $r_{\mathrm{m}}$ before 1958 become rising trends since 1958, and the weakly rising trend in $L_{\mathrm{m}}$ before 1958 seems to disappear since 1958 . This may imply a periodicity longer than 140 years, probably related to a possible double Gleissberg cycle or the 200-year Suess cycle. The local minima in the correlations $\left(r_{0}\right.$ and $\left.r_{\mathrm{m}}\right)$ around 1958 are related to the increasing trend in $R_{\mathrm{Z}}$ before 1958, a possible decreasing trend since then, and the non-linearity between $R_{\mathrm{Z}}$ and $a a$ (see also Du, 2011b).

An odd-numbered solar cycle tends to show a higher correlation $\left(r_{\mathrm{n} 0}\right.$ or $\left.r_{\mathrm{nm}}\right)$ and a shorter lag time $\left(L_{\mathrm{nm}}\right)$ between $R_{\mathrm{z}}$ and $a a$ than the previous even-numbered one, suggesting a 2-cycle periodicity superimposed on secular trends (Fig. 3). An even-numbered Hale cycle tends to show a higher correlation $\left(r_{\mathrm{H} 0}\right.$ or $\left.r_{\mathrm{Hm}}\right)$ and a shorter lag time $\left(L_{\mathrm{Hm}}\right)$ between $R_{\mathrm{Z}}$ and $a a$ than the previous odd-numbered one, suggesting a 4-cycle periodicity superimposed on secular trends (Fig. 4). However, the 5-cycle (in $r_{\mathrm{n} 0}$ ) and 8-cycle (in $r_{\mathrm{D} 0}$ ) periodicities suggested by Borello-Filisetti et al. (1992) and Mussino et al. (1994) have not been found in this study.

Around a solar cycle maximum $a a$ tends to lag behind $R_{\mathrm{z}}$ about 2-3 years (Wang et al., 2000; Echer et al., 2004), while around a cycle minimum the lag time is small, at about 1 year (Legrand and Simon, 1981; Wilson, 1990; Wang and Sheeley, 2009), the reason for which will be discussed in the following paper (Du, 2011b). This can roughly explain the stronger correlation at the ascending phase and the weaker correlation at the descending phase. At the ascending phase of a solar cycle, the solar activity affects the geomagnetic activity usually in a simple(r) way. With the increase of solar activity, the geomagnetic activity (especially the transient part) increases accordingly, and thus $r_{\mathrm{A}}$ is positive, strong and almost stable ( $\sim 0.48$; see Fig. $3 a)$. This correlation is nearly unaffected by the time delay of $a a$ to $R_{\mathrm{z}}$ because the declining tail of $a a$ in the preceding cycle and the lag time near the onset of the current cycle are both small(er). The time delay of $a a$ after the maximum of the cycle affects the descending part rather than the ascending one.

At the descending phase of a solar cycle, however, the time delay of $a a$ (usually the recurrent part) affects the correlation greatly. When $R_{\mathrm{z}}$ has already begun to decrease after its maximum, $a a$ is still increasing as a consequence of the time delay response of the rising activity during the later part of the preceding ascending phase. The opposite changes in $a a$ and in $R_{\mathrm{z}}$ can partly cancel the originally positive correlation between $a a$ and $R_{\mathrm{z}}$ (when $a a$ follows the declining $R_{\mathrm{Z}}$ ). Therefore, the correlation at the descending phase $\left(\bar{r}_{\mathrm{D} 0}=0.24\right)$ is in general weaker than that at the ascending phase $\left(\bar{r}_{\mathrm{A} 0}=0.48\right)$, while it is unaffected by the lag tail of $a a$ entering into the next cycle.

The solar activity affects the geomagnetic activity in a much more complex way at descending phases than at ascending phases. The geomagnetic activity does not vary linearly with the solar activity, with more peaks usually present at descending phases than at ascending phases (Echer et al., 2004; Wang and Sheeley, 2009). The complex physical mechanisms and different physical conditions result in variations in the levels and phases of $a a$, which lead to the different correlations and time delays of $a a$ to $R_{\mathrm{Z}}$ for different cycles (Fig. 3). 
Legrand and Simon (1989) classified the geomagnetic activity ( $a a$ index) in four classes related to solar activity: (1) the magnetic quiet activity due to slow solar wind flowing around the magnetosphere, (2) the recurrent activity related to high wind speed solar wind, (3) the fluctuating activity related to fluctuating solar wind and (4) the shock activity due to shock events (CME). These activities peak at different times relative to the peak of sunspot cycle. The variation in the correlation between $R_{\mathrm{Z}}$ and $a a$ is due to their different heliospheric sources. $R_{\mathrm{Z}}$ is a proxy that represents the solar surface magnetic activity, while $a a$ integrates the effects on magnetosphere of several other sources, such as solar flares, coronal mass ejections (CMEs), and fast solar wind streams. The solar transient activity (e.g. solar flares) dominates the ascending phases (Borello-Filisetti et al., 1992), while recurrent geomagnetic activity is more frequent during the declining phase or at the minimum of the solar cycle (Sargent, 1985) and is commonly associated with high-speed solar wind streams emanating from coronal holes, corotating with the Sun (Bame et al., 1976; Legrand and Simon, 1989).

The decreasing trend in the correlation between $R_{\mathrm{Z}}$ and $a a$ has been explained by the increasing occurrence of highspeed solar wind streams during the descending phase of solar cycle (Bame et al., 1976; Borello-Filisetti et al., 1992; Mussino et al., 1994; Tsurutani et al., 1995; Kishcha et al., 1999). Echer et al. (2004) suggested that the probable cause of the correlation decrease seems to be related to the dual peak structure of the $a a$ index with the increase of the second $a$ a peak. However, the long-term decreasing trend in the correlation is not exclusively caused by the increasing trend in the lag time; it is still decreasing even after considering the effect of the lag time (see $r_{\mathrm{m}}$ in Fig. 2). The causes of the decreasing trend in the correlation between $R_{\mathrm{z}}$ and $a a$ and the increasing trend in the lag time of $a a$ to $R_{\mathrm{Z}}$ may be related to the increase in solar magnetic activity over the last century (Lockwood et al., 1999). These phenomena and the reason for the increasing occurrence of high-speed solar wind streams during the descending phase of solar cycle will be further discussed in the following paper (Du, 2011b).

The variations in $R_{\mathrm{Z}}$ and $a a$ and hence in their correlation are related to the Hale and other long-term cycles, such as 55-, 80-, 200- and 1000-year cycles (Jose, 1965; Yoshimura, 1979; Feynman and Gabriel, 1990; Borello-Filisetti et al., 1992; Landscheidt, 1999; Du, 2006a,b; Demetrescu and Dobrica, 2008). Therefore, the correlation cannot decrease infinitely. The 2-cycle periodicity in $r_{\mathrm{nm}}$ or $L_{\mathrm{nm}}$ reflects the asymmetry of the solar cycle, the so-called G-O rule: an oddnumbered cycle tends to be stronger than the previous evennumbered one (Gnevyshev and Ohl, 1948; Wilson, 1988). The stronger correlations for odd-numbered cycles than for even-numbered cycles reflect the G-O rule and the less decays for odd-numbered cycles than for even-numbered cycles (Du, 2011a). Stamper et al. (1999) noted that the solar wind speed peaks strongly in the declining phase of evennumbered cycles and can be identified as the chief cause of the phase shift between the sunspot numbers and the aa index. The variations in the correlations for solar cycles may be also related to the non-linearity between $R_{\mathrm{Z}}$ and $a a$. For example, the average ratios of $a a / R_{\mathrm{Z}}$ during the three years around the peaks of sunspot Cycles 18-20 are 0.23, 0.16 and 0.20 , respectively. It implies that the generation efficiency of geomagnetic activity by solar activity in a stronger cycle (19) tends to be lower than that in a weaker cycle $(18,20)$. The 4-cycle periodicity in $r_{\mathrm{H} 0}, r_{\mathrm{Hm}}$ and $L_{\mathrm{Hm}}$ reflects the asymmetry of the Hale cycle: the correlation between $R_{\mathrm{z}}$ and $a a$ for an even-numbered $H$-cycle tends to be stronger than the previous odd-numbered one (Fig. 4). If the Hale cycle number $(H)$ is decreased (or increased) by one, $H^{\prime}=H-1$, then the statement is similar to that for the $\mathrm{G}-\mathrm{O}$ rule: an oddnumbered Hale cycle $\left(H^{\prime}\right)$ tends to have a stronger correlation between $R_{\mathrm{Z}}$ and $a a$ than the previous even-numbered one. This cycle may reflect the 44-year cycle existed in $R_{\mathrm{Z}}$ (Du et al., 2009) and sunspot area (Javaraiah, 2008). A periodicity longer than 140 years as suggested in Fig. 2 reflects the long-term periodicities of double Gleissberg cycles (Usoskin and Mursula, 2003; Du, 2006a; Du and Du, 2006), 179-year (Jose, 1965; Landscheidt, 1999), the well known 200-year Suess cycle etc., and their asymmetries as well.

To study the correlation between the solar and geomagnetic activity is important for understanding the physical mechanism of the solar cycle. Dynamo models can reproduce certain features of the 11-year cycle but cannot explain the varying amplitudes of maxima and other long-term changes (Eddy, 1976; Javaraiah et al., 2005), and their predictive power needs to be checked in the future (Schatten, 2005; Dikpati et al., 2006; Choudhuri et al., 2007; Bushby and Tobias, 2007). The weakening trend of the correlation and its long-term cycle variations may affect the theory of solar dynamo models for explaining the phenomena of the solar cycle. In fact, in the next paper (Du, 2011b), I will present a model that could bring a natural explanation to the above phenomena.

At last, we examine whether the above conclusions are affected by the possible error in $a a$ before 1957 (Svalgaard et al., 2004; Nevanlinna and Kataja, 1993; Svalgaard and Cliver, 2007; Lukianova et al., 2009). If the original uncorrected $a a$ series were used, there would have not been significant changes both in the $r$-coefficient at $L=0\left(r_{0}=0.45\right.$ from 0.43$)$ and in the maximum one $\left(r_{\mathrm{m}}=0.49\right.$ from 0.47$)$ which is at the same lag $L_{\mathrm{m}}=15$ in Fig. $1 \mathrm{~b}$. This is due to the evenly distribution of the correction in $a a$ before 1957 .

The results in Fig. 2 are re-analyzed when using the uncorrected $a a$ series, as shown in Fig. 5. One can see in this figure that there are some large negative jumps in $L_{\mathrm{m}}(\sim-40)$ during September 1957-October 1958 and one in August 1966. It may be caused by the inconsistent in the $a a$ series due to the station inter-calibration in 1957 when the northern $a a$ station was changed from Abinger to Hartland (Svalgaard and Cliver, 2007; Lukianova et al., 2009). 


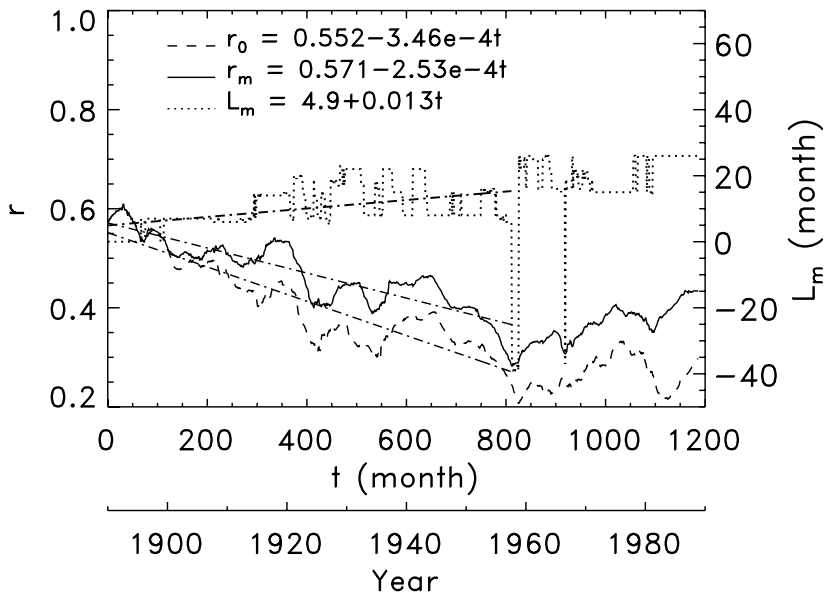

Fig. 5. Similar to Fig. 2 using the uncorrected $a a$ series.

However, there are no significant changes in the trends in $r_{0}, r_{\mathrm{m}}$ and $L_{\mathrm{m}}$ before August 1957,

$$
\begin{aligned}
& r_{0}=0.552 \pm 0.003-(3.46 \pm 0.05) \times 10^{-4} t, \\
& r_{\mathrm{m}}=0.571 \pm 0.002-(2.53 \pm 0.04) \times 10^{-4} t, \\
& L_{\mathrm{m}}=4.9 \pm 0.4-(0.013 \pm 0.001) t
\end{aligned}
$$

The standard deviations of $r_{0}$ and $r_{\mathrm{m}}\left(\sigma_{0}=0.036\right.$ and $\sigma_{\mathrm{m}}=$ $0.030)$ are slightly larger than those $(0.029$ and 0.026$)$ in Fig. 2, and the fitting correlation coefficients $\left(r_{\mathrm{f}}=0.91\right.$ and $0.89)$ are slightly lower than those (0.96 and 0.95) in Fig. 2. The standard deviation of $L_{\mathrm{m}}\left(\sigma_{\mathrm{L}}=5.4\right)$ is slightly smaller than that (5.8) in Fig. 2, and the fitting correlation coefficient $\left(r_{\mathrm{f}}=0.49\right)$ is slightly higher than that $(0.41)$ in Fig. 2.

In addition, the results in Figs. 3-4 (Sects. 3-4) have none or little changes if using the uncorrected $a a$ series. Therefore, correcting the possible error in $a a$ before 1957 is reasonable from the above results, while it does affect the general conclusions in the present study.

According to the analysis above, main conclusions are summarized as follows.

1. The correlation $\left(r_{0}\right)$ at zero lag, the maximum $\left(r_{\mathrm{m}}\right)$ of lag-correlation function and the lag time $\left(L_{\mathrm{m}}\right)$ between the solar and geomagnetic activities for a 528-month running time window vary in a declining, declining and rising secular trend, respectively, before 1958. However, these trends changed: both $r_{0}$ and $r_{\mathrm{m}}$ vary in rising secular trends and there is not a significant trend in $L_{\mathrm{m}}$ since 1958, probably related to a periodicity longer than 140 years.

2. An odd-numbered solar cycle tends to show a higher correlation $\left(r_{\mathrm{n} 0}\right.$ or $\left.r_{\mathrm{nm}}\right)$ and a shorter lag time $\left(L_{\mathrm{nm}}\right)$ between $R_{\mathrm{Z}}$ and $a a$ than the previous even-numbered one, suggesting a 2-cycle periodicity superimposed on secular trends.
3. An even-numbered Hale cycle tends to show a higher correlation $\left(r_{\mathrm{H} 0}\right.$ or $\left.r_{\mathrm{Hm}}\right)$ and a shorter lag time $\left(L_{\mathrm{Hm}}\right)$ between $R_{\mathrm{Z}}$ and $a a$ than the previous odd-numbered one, suggesting a 4-cycle periodicity superimposed on secular trends.

4. The variations in the correlations may be related to the non-linearity between $R_{\mathrm{z}}$ and $a a$, and the decreasing trend in the correlation $\left(r_{0}\right)$ is not exclusively caused by the increasing trend in the lag time of $a a$ to $R_{\mathrm{Z}}$.

Acknowledgements. We are grateful to the referees for suggestive and helpful comments. This work is supported by National Natural Science Foundation of China (NSFC) through grants 10973020, 40890161 and 10921303, and National Basic Research Program of China through grants 2011CB811406.

Topical Editor P. Drobinski thanks L. Damé and two other anonymous referees for their help in evaluating this paper.

\section{References}

Bame, S. J., Asbridge, J. R., Feldman, W. C., and Gosling, J. T.: Solar cycle evolution of high-speed solar wind streams, Astrophys. J., 207, 977-980, 1976.

Borello-Filisetti, O., Mussino, V., Parisi, M., and Storini, M.: Longterm variations in the geomagnetic activity level. I - A connection with solar activity, Ann. Geophys., 10, 668-675, 1992.

Bushby, P. J. and Tobias, S. M.: On Predicting the Solar Cycle Using Mean-Field Models, Astrophys. J., 661, 1289-1296, 2007.

Cameron, R. and Schüssler, M.: Solar Cycle Prediction Using Precursors and Flux Transport Models, Astrophys. J., 659, 801-811, 2007.

Chernosky, E. J.: Double Sunspot-Cycle Variation in Terrestrial Magnetic Activity 1884-1963, J. Geophys. Res., 71, 965-974, 1966.

Choudhuri, A. R., Chatterjee, P., and Jiang, J.: Predicting Solar Cycle 24 With a Solar Dynamo Model, Phys. Rev. Lett., 98, 131103, doi:10.1103/PhysRevLett.98.131103, 2007.

Clilverd, E. W., Boriakoff, V., and Feynman, J.: Solar variability and climate change: Geomagnetic aa index and global surface temperature, Geophys. Res. Lett., 25, 1035-1038, 1998.

Demetrescu, C. and Dobrica, V.: Signature of Hale and Gleissberg solar cycles in the geomagnetic activity, J. Geophys. Res., 113, A02103, doi:10.1029/2007JA012570, 2008.

Dikpati, M., de Toma, G., and Gilman, P. A.: Predicting the strength of solar cycle 24 using a flux-transport dynamo-based tool, Geophys. Res. Lett., 33, L05102, doi:10.1029/2005GL025221, 2006.

Du, Z. L.: Relationship Between Solar Maximum Amplitude and Max-Max Cycle Length, Astron. J., 132, 1485-1489, 2006 a.

Du, Z. L.: A new solar activity parameter and the strength of 5-cycle periodicity, New Astronomy, 12, 29-32, 2006b.

Du, Z. L.: The correlation between solar and geomagnetic activity - Part 1: Two-term decomposition of geomagnetic activity, Ann. Geophys., Ann. Geophys., 29, 1331-1340, doi:10.5194/angeo29-1331-2011, 2011a.

Du, Z. L.: The correlation between solar and geomagnetic activity - Part 3: An integral response model, Ann. Geophys., 29, 10051018, doi:10.5194/angeo-29-1005-2011, $2011 \mathrm{~b}$. 
Du, Z. and Du, S.: The Relationship between the Amplitude and Descending Time of a Solar Activity Cycle, Solar Phys., 431437, 2006.

Du, Z. L. and Wang, H. N.: Is a higher correlation necessary for a more accurate prediction?, Science China (Physics, Mechanics \& Astronomy), 54, 172-175, 2011.

Du, Z. L., Li, R., and Wang, H. N.: The Predictive Power of Ohl's Precursor Method, Astron. J., 138, 1998-2001, 2009.

Duhau, S.: An Early Prediction of Maximum Sunspot Number in Solar Cycle 24, Solar Phys., 213, 203-212, 2003.

Echer, E., Gonzalez, W. D., Gonzalez, A. L. C., Prestes, A., Vieira, L. E. A., dal Lago, A., Guarnieri, F. L., and Schuch, N. J.: Longterm correlation between solar and geomagnetic activity, J. Atmos. Sol. Terr. Phys., 66, 1019-1025, 2004.

Eddy, J. A.: The Maunder Minimum, Science, 192, 1189-1202, 1976.

Feynman, J.: Geomagnetic and solar wind cycles, 1900-1975, J. Geophys. Res., 87, 6153-6162, 1982.

Feynman, J. and Crooker, N. U.: The solar wind at the turn of the century, Nature, 275, 626-627, 1978.

Feynman, J. and Gabriel, S. B.: Period and phase of the 88-year solar cycle and the Maunder minimum - Evidence for a chaotic sun, Solar Phys., 127, 393-403, 1990.

Gnevyshev, M. N. and Ohl, A. I.: On the 22-year solar activity cycle, Astron. Z., 25, 18-20, 1948.

Javaraiah, J.: Predicting the Amplitude of a Solar Cycle Using the North-South Asymmetry in the Previous Cycle: II. An Improved Prediction for Solar Cycle 24, Solar Phys., 252, 419-439, 2008.

Javaraiah, J., Bertello, L., and Ulrich, R. K.: Long-Term Variations in Solar Differential Rotation and Sunspot Activity, Solar Phys., 232, 25-40, 2005.

Jose, P. D.: Sun's motion and sunspots, Astron. J., 70, 193-200, 1965.

Kishcha, P. V., Dmitrieva, I. V., and Obridko, V. N.: Long-term variations of the solar-geomagnetic correlation, total solar irradiance, and northern hemispheric temperature (1868-1997), J. Atmos. Sol. Terr. Phys., 61, 799-808, 1999.

Landscheidt, T.: Extrema in Sunspot Cycle Linked to Sun's Motion, Solar Phys., 189, 413-424, 1999.

Legrand, J. P. and Simon, P. A.: Ten cycles of solar and geomagnetic activity, Solar Phys., 70, 173-195, 1981.

Legrand, J. P. and Simon, P. A.: Solar cycle and geomagnetic activity: A review for geophysicists. I - The contributions to geomagnetic activity of shock waves and of the solar wind, Ann. Geophys., 7, 565-578, 1989.

Lockwood, M., Stamper, R., and Wild, M. N.: A doubling of the Sun's coronal magnetic field dring the past 100 years, Nature, 399, 437-439, 1999.

Lukianova, R., Alekseev, G., and Mursula, K.: Effects of station relocation in the aa index, J. Geophys. Res., 114, A02105, doi:10.1029/2008JA013824, 2009.

Mayaud, P. N.: The aa indices: A 100-year series characterizing the magnetic activity, J. Geophys. Res., 77, 6870-6874, 1972.

Mursula, K., Martini, D., and Karinen, A.: Did open solar magnetic field increase during the last 100 years? A reanalysis of geomagnetic activity, Solar Phys., 224, 85-94, 2004.
Mussino, V., Borello Filisetti, O., Storini, M., and Nevanlinna, H.: Long-term variations in the geomagnetic activity level Part II: Ascending phases of sunspot cycles, Ann. Geophys., 12, 10651070, doi:10.1007/s00585-994-1065-5, 1994.

Nevanlinna, H. and Kataja, E.: An extension of the geomagnetic activity index series aa for two solar cycles (1844-1868), Geophys. Res. Lett., 20, 2703-2706, 1993.

Oliver, R., Ballester, J. L., and Baudin, F.: Emergence of magnetic flux on the Sun as the cause of a 158-day periodicity in sunspot areas, Nature, 394, 552-553, 1998.

Prestes, A., Rigozo, N. R., Echer, E., and Vieira, L. E. A.: Spectral analysis of sunspot number and geomagnetic indices (18682001), J. Atmos. Sol. Terr. Phys., 68, 182-190, 2006.

Russell, C. T. and Mulligan, T.: The 22-year variation of geomagnetic activity: Implications for the polar magnetic field of the Sun, Geophys. Res. Lett., 22, 3287-3288, 1995.

Sargent, H. H.: Recurrent geomagnetic activity - Evidence for long-lived stability in solar wind structure, J. Geophys. Res., 90, 1425-1428, 1985.

Schatten, K. H.: Fair space weather for solar cycle 24, Geophys. Res. Lett., 32, L21106, doi:10.1029/2005GL024363, 2005.

Schatten, K. H., Scherrer, P. H., Svalgaard, L., and Wilcox, J. M.: Using dynamo theory to predict the sunspot number during solar cycle 21, Geophys. Res. Lett., 5, 411-414, 1978.

Stamper, R., Lockwood, M., Wild, M. N., and Clark, T. D. G.: Solar causes of the long-term increase in geomagnetic activity, J. Geophys. Res., 104, 28325-28342, 1999.

Svalgaard, L. and Cliver, E. W.: Interhourly variability index of geomagnetic activity and its use in deriving the long-term variation of solar wind speed, J. Geophys. Res., 112, A10111, doi:10.1029/2007JA012437, 2007.

Svalgaard, L., Cliver, E. W., and Le Sager, P.: IHV: a new long-term geomagnetic index, Adv. Space. Res., 34, 436-439, 2004.

Tsurutani, B. T., Gonzalez, E. D., Gonzalez, A. L. C., Tang, F., Arballo, J. K., and Okada, M.: Interplanetary origin of geomagnetic activiy in the declining phase of the solar cycle, J. Geophys. Res., 21, 717-734, 1995.

Usoskin, I. G. and Mursula, K.: Long-Term Solar Cycle Evolution: Review of Recent Developments, Solar Phys., 218, 319343, 2003.

Wang, Y. M. and Sheeley, N. R.: Understanding the Geomagnetic Precursor of the Solar Cycle, Astrophys. J., 694, L11-L15, 2009.

Wang, Y. M., Lean, J., and Sheeley, N. R.: The long-term variation of the Sun's open magnetic flux, Geophys. Res. Lett., 27, 505508, 2000.

Wilson, R. M.: Bimodality and the Hale cycle, Solar Phys., 117, 269-278, 1988.

Wilson, R. M.: On the level of skill in predicting maximum sunspot number - A comparative study of single variate and bivariate precursor techniques, Solar Phys., 125, 143-155, 1990.

Yoshimura, H.: The solar-cycle period-amplitude relation as evidence of hysteresis of the solar-cycle nonlinear magnetic oscillation and the long-term $/ 55$ year/ cyclic modulation, Astrophys. J., 227, 1047-1058, 1979. 